\title{
Development of a Real-time Framework between MATLAB and PLC through OPC-UA: A case study of a microgrid energy management system
}

Abdallah EL ZERK ( $\sim$ abdallahelzerk@research.emi.ac.ma )

Mohammed V University of Rabat Mohammadia School of Engineering: Universite Mohammed V de

Rabat Ecole Mohammadia d'Ingenieurs https://orcid.org/0000-0003-4940-0526

Mohammed OUASSAID

Mohammed V University of Rabat Mohammadia School of Engineering: Universite Mohammed V de Rabat Ecole Mohammadia d'Ingenieurs

\section{Youssef ZIDANI}

Cadi Ayyad University Faculty of Science and Technology Gueliz: Universite Cadi Ayyad Faculte des Sciences et Techniques Gueliz

\section{Research Article}

Keywords: Real-Time simulation, PLC, OPC-UA, Renewable Energy System (RES), Energy Management Systems (EMS), Fuzzy Logic Controller (FLC)

Posted Date: January 26th, 2022

DOI: https://doi.org/10.21203/rs.3.rs-1265626/v1

License: (9) This work is licensed under a Creative Commons Attribution 4.0 International License. Read Full License 


\title{
Development of a Real-time Framework between MATLAB and PLC through OPC-UA: A case study of a microgrid energy management system
}

\author{
Abdallah EL ZERK ${ }^{1 *}$, Mohammed OUASSAID ${ }^{1}$, Youssef ZIDANI $^{2}$ \\ ${ }^{1}$ Engineering for Smart and Sustainable Systems Research Centre, Mohammadia School of Engineers, Mohammed V University \\ in Rabat, Rabat, Morocco. \\ ${ }^{2}$ Department of Electrical Engineering Faculty of science and technology, Cadi Ayyad University, Morocco \\ Abdallahelzerk@,research.emi.ac.ma, ouassaid@emi.ac.ma, zidani@yahoo.com
}

\begin{abstract}
Many research laboratories, which develop advanced algorithms for controlling Renewable Energy Systems (RES), lack specialized equipment to carry out research activities and perform experiments. Real-time simulation platforms are suitable for designing specific applications such as fast or real-time computation models. In addition, these platforms provide fast, accurate and reliable studies for RES control implementation. However, Real-time platforms are too expensive and not affordable for most laboratories. In this context, a cost-effective platform is designed to assist researchers in their activities. The hardware solution consists of using a Programmable Logic Control (PLC) to acquire data from the various sensors and control the system through actuators and PID controllers. From a software standpoint, a communication framework between MATLAB and the PLC is developed using the OPCUA (Open Platform Communications Unified Architecture) technology. In this paper, the operation and efficiency of the platform have been tested using the Software in the Loop (SIL) technique. Hence, the operating model is designed using MATLAB/Simulink to simulate and analyze the appropriate control technique suitable for PLC implementation, whereas the control is executed through Siemens S71200 PLC using TIA PORTAL. A visualization interface is set up using WinCC-RT. Furthermore, this paper describes the design of the platform and discusses its utilization, providing examples of its usage through a case study. Indeed, a complex algorithm developed in MATLAB/Simulink can be automatically executed by any PLC using the designed framework.
\end{abstract}

Keywords-Real-Time simulation, PLC, OPC-UA, Renewable Energy System (RES), Energy Management Systems (EMS), Fuzzy Logic Controller (FLC).

Conflict-of-interest statement: The authors have no conflicts of interest to declare.

\section{INTRODUCTION}

\section{A. Context and problematic}

Recently, distributed generation and renewable energy systems are attracting more and more attention due to environmental issues. Between global warming induced by greenhouse gases and the criticality of the risk posed by nuclear power plants, which presented themselves as an alternative to fossil fuels, the world is faced with the question of the energy future, particularly with regard to electricity production. Following the rapid growth of the energy industry segment and the complexity introduced by the control of RES, there is a need to develop an advanced engineering tool to model, design, simulate, monitor, control and implement these systems [1].

More recently, researchers are increasingly interested in high-level programming languages, such as MATLAB, Mathcad, Mathematica, etc. These platforms can be exploited to model, design and analyze the RES behavior. Among these platforms, MATLAB has been trusted to be used for a variety of tasks ranging from economics to the sciences [2].

A research work is more interesting and consistent if it is supported by experimental validation. To do this, the researcher must have the possibility to:

- Design and validate the developed algorithms in MATLAB which happens to be the most common platform used by most researchers nowadays - without the need to use other tools.

- Practice through programming boards that are firstly within the researcher's reach and secondly support MATLAB.

In this paper, a low-cost solution that allows the researcher to easily perform experiments under MATLAB is proposed. This solution does not require the deployment of expensive programming cards.

\section{B. Interoperability issues}

In the engineering discipline, several types of control strategies can be designed through MATLAB/Simulink software. Fuzzy 
logic (FL), neural networks (NN), genetic algorithms (GA) and multi-agent systems (MAS) are the most commonly used for RES's energy management $[3,4]$. Indeed, system simulation and management algorithm design are within the reach of the researcher thanks to the MATLAB environment. However, their practical implementation is a difficult task and relatively expensive.

In order to implement an algorithm, the researcher can use programming and processing boards such as DSP, DSPACE, FPGA, Microcontroller [5,6,7]. Indeed, due to the incompatibility with MATLAB and interoperability issues, it is necessary to use other proprietary programming platforms to implement the developed algorithms.

The programmable logic controller (PLC) designed to perform logic functions has been widely employed in various process industries $[8,9,10]$. The PLC's use offers several advantages, including flexibility, reliability, low power consumption and ease of expansion. In the last decade, the major PLC manufacturers have made significant improvements, notably through the introduction of OPC (Open Platform Communications) technology [11,12,13,14]. The recent development of this protocol has promoted interoperability between several environments. Furthermore, since OPC technology has been adopted by the leading PLC manufacturer Siemens [15], a connection between the Siemens-based PLC and the MATLAB environment is now possible.

\section{OPC-UA technology}

Standardizing a communication language overcomes the software and hardware communication challenges. As already stated, MATLAB and programming boards, even though they have been designed by different companies, have no reason to operate separately, as the value is created by their interaction and exchange of information.

This is where the OPC-UA standard comes into play by facilitating the exchange and management of data to meet all the requirements of researchers today, including the ability to perform experiments. Indeed, the adoption of a unified and open-source communication protocols as OPC-UA is the next step to develop a framework between MATLAB and Siemens PLC. OPC-UA is an open standard defined as a set of objects, interfaces and methods to facilitate interoperability between different environments. Furthermore, from a cybersecurity perspective, OPC-UA allows digitally signed and encrypted information to be exchanged within a network using advanced algorithms [16]. The variability and flexibility of OPC-UA range from simple process data acquisition to complex monitoring, control, and analysis. In this paper, the OPC-UA protocol is utilized to enable reliable communication between MATLAB and the PLC.

\section{Related works}

Some works have been carried out worldwide to help research activities and perform experiments on microgrids. In [17], an experimental validation procedure of a simple cascade control system through several architectures, such as SCADA, PLC and OPC is performed. In this study, PID is implemented on MicroLogix-1200 PLC and RSView-32 SCADA has been used with RSLinx communication software. The work in [18] presents a house intelligent management system. The developed algorithm has been performed using real-time simulation from Opal-RT. This makes the integration of MATLAB/Simulink and real-time simulation models possible. The decentralized control of DC microgrids using hybrid renewable energy sources and batteries which operate with and without grid-connected mode is proposed in [19]. The proposed model is developed with MATLAB/Simulink SimPowerSystem and simulated with real-time simulation using OPAL-RT.

\section{E. Contribution}

The research presented in this paper brings important contributions in the engineering research field, such as renewable energy control, embedded systems and sustainable development. By combining the Siemens ecosystem power which supports the OPCUA protocol and TIA PORTAL (Totally Integrated Automation), it is now possible to develop any algorithm in MATLAB and implement it through a PLC. This helps researchers to easily execute developed algorithms using MATLAB through PLCs. The main contribution of this article lies in the following aspects:

- Designing a cost-effective solution, helping researchers to perform experiments on Artificial Intelligence (AI) algorithms.

- Developing a communication framework between MATLAB, PLC and Human Machine Interface (HMI) using an OPCUA technology.

- Validation of developed framework through a case study in research field. Indeed, an advanced energy management system (EMS) based on a fuzzy logic controller to enhance power quality and load supply within a microgrid is applied.

- Designing an HMI for monitoring the microgrid using WinCC-RT.

\section{F. Structure of the paper}

The general paper organization is as follows. In Section II, the system architecture is detailed. Firstly, the overall control scheme is described. Secondly, the communication between MATLAB, PLC and HMI is developed. Section III describes the EMS-based fuzzy logic implementation. Section V outlines the conclusion.

\section{SYSTEM ARCHITECTURE}

In an OPC-UA communication structure where the server and the client are involved, the server responds to client requests by transferring data based on the client demands. OPC clients are supposed to be used on the application side to communicate with 
an appropriate OPC server. MATLAB supports OPC-UA communication and can establish it [20].

Indeed, it is possible to connect MATLAB as a client to a server that is OPC-UA compliant and read/write data through it. Fig. 1 shows the structure of the proposed OPC-UA connection.

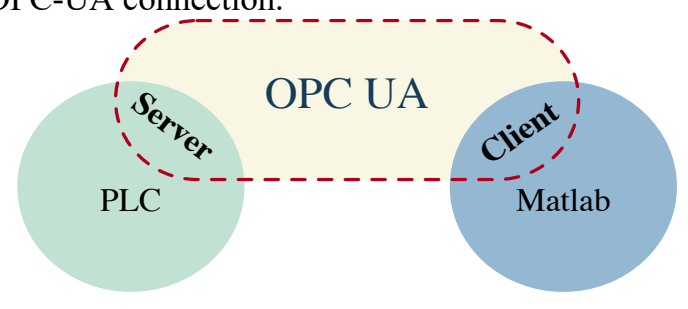

Fig. 1. Structure of the proposed OPC-UA.

\section{A. Overall control scheme}

As presented in Fig. 2, the structure of the proposed platform consists of three main parts:

- The workstation where MATLAB and TIA PORTAL are installed.

- The PLC, which in this paper is simulated using PLCSim.

- Operating model which represents the studied microgrid.

The workstation includes the programming tools, namely MATLAB and TIA PORTAL in conjunction with the OPC-UA software. This latter allows efficient communication between MATLAB and the PLC. MATLAB provides AI capabilities similar to those of dedicated AI tools. Furthermore, it permits algorithm integration into the complete workflow for developing a fully engineered system. In this paper, MATLAB is deployed to design, optimize, simulate and validate the AI algorithm. Then, the control result is transferred into the PLC through the OPC server to be executed.

Designed for high efficiency and user-friendliness, TIA Portal is an engineering framework created to program Siemens devices

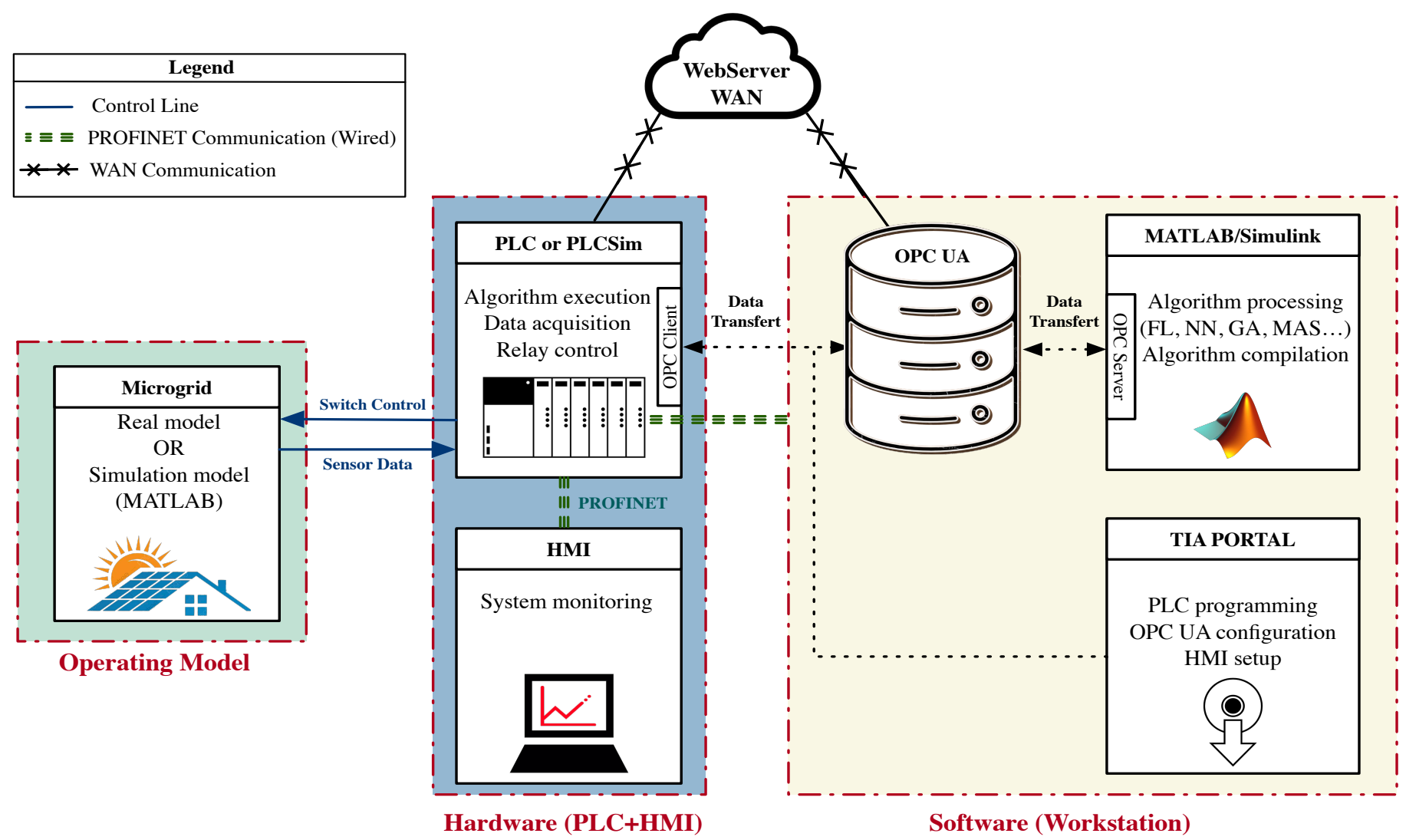

Fig. 2. Structure of the designed platform.

[21]. The software engineering framework enables users to develop and commission automation systems quickly and intuitively, which eliminates the traditional time consuming and costly integration of separate software packages.

The PLC ensures the following functions:

- Data acquisition from various devices and sensors such as current, voltage and temperature,

- Actuator's control such as solid-state relays,

- Algorithm's execution. 
The PLC used in this study is Siemens S7-1214C DC/DC/DC controller. The considered PLC has various unique features such as an HMI connection possibility, reduced system cost and the support of OPC-UA technology. The PLC is programmed using ladder language according to IEC 61131-3 standard [22].

An HMI is developed using WinCC software [23], which has functions of parameter setting, status display, remote control and data storage. The HMI is created to display process variables such as battery State of Charge (SoC), power consumption and generation, load profile and climate conditions. The developed interface can also store data for further utilization.

The framework developed in this study allows control strategies to be executed through the following techniques [24]:

- Software in the Loop (SIL): Using the same workstation, both the operating model and the algorithm are running through MATLAB. However, the PLC and PID are simulated using PLCSim under

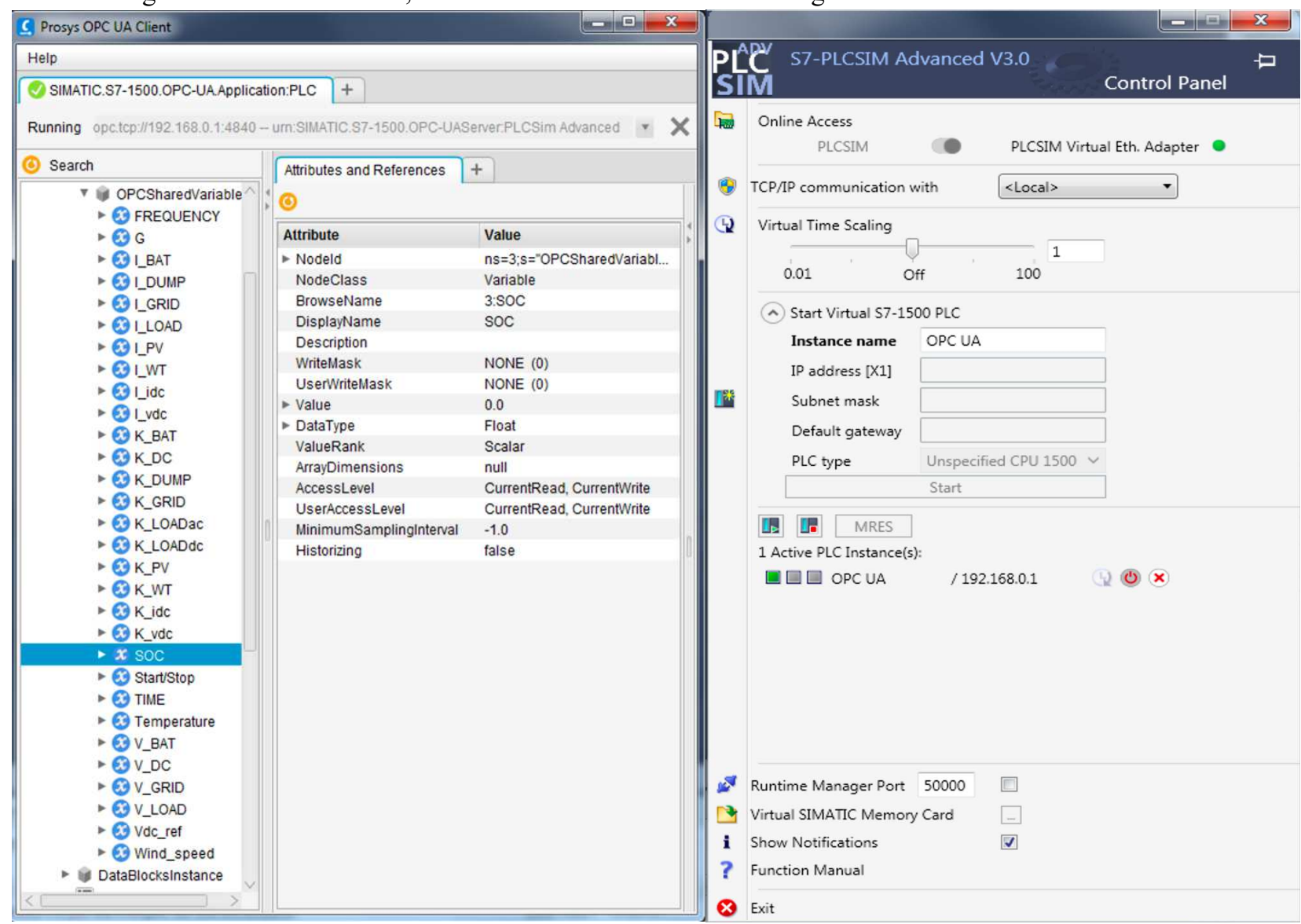

Fig. 3. Setting up communication between Prosys OPC UA and PLCSim via TCP/IP protocol.

- TIA PORTAL. The simulation includes the electrical emulation of sensors and actuators. These electrical emulations allow interfacing between the plant simulation and the embedded system under the test.

- Processor in the Loop (PIL): The operating model

- is simulated under MATLAB while the algorithm is executed using a real PLC.

- Hardware in the Loop (HIL): The algorithm is executed on the workstation. The operating model and the controller are performed in real-time systems.

Table I describes all the hardware components and software tools used in this study.

TABLE I

Hardware Components and Software Platforms used in The System

\begin{tabular}{cccc}
\hline Type & Name & Description & Reference \\
\hline \multirow{3}{*}{ Hardware } & CPU 1214C AC/DC/RLY & Siemens PLC & 6ES7 214-1BG4O-0XB0 \\
\cline { 2 - 4 } & AI 8x13bit & Analog input module & 6ES7 231-4HF32-0XB0 \\
\cline { 2 - 4 } & AQ 4x14bit & Analog output module & 6ES7 232-4HD30-0XB0 \\
\hline \multirow{2}{*}{ Software } & MATLAB 2019 & &
\end{tabular}




\author{
WinCC Professional V15.1 \\ PLCSim Adv V3 \\ Prosys OPC UA Client
}

\title{
B. Establishment of MATLAB-HMI-PLC connection
}

In this part, a method to realize the communication between PLC and MATLAB is introduced. The two environments are heterogeneous since they are developed by two different companies namely MathWorks and Siemens. Indeed, PLC and MATLAB cannot communicate directly. However, OPC-UA technology can be exploited to ensure the appropriate communication between PLC and MATLAB.

\section{1) Establishment of MATLAB-PLC connection}

In this work, the PLC configured as an OPC server communicates with MATLAB regarded as an OPC client. During the setup of the connection, the OPC server creates a visible configurable path to the OPC client to share variables. The path creates the possibility of accessing all the available registers in the PLC from any OPC client software.

Under the TIA PORTAL environment, a DataBlock (DB) should be created to connect PLC variables with the OPC server. In the meantime, a static IP address must be assigned to the PLC in order to establish a connection with the Prosys OPC- UA as shown in Fig. 3.

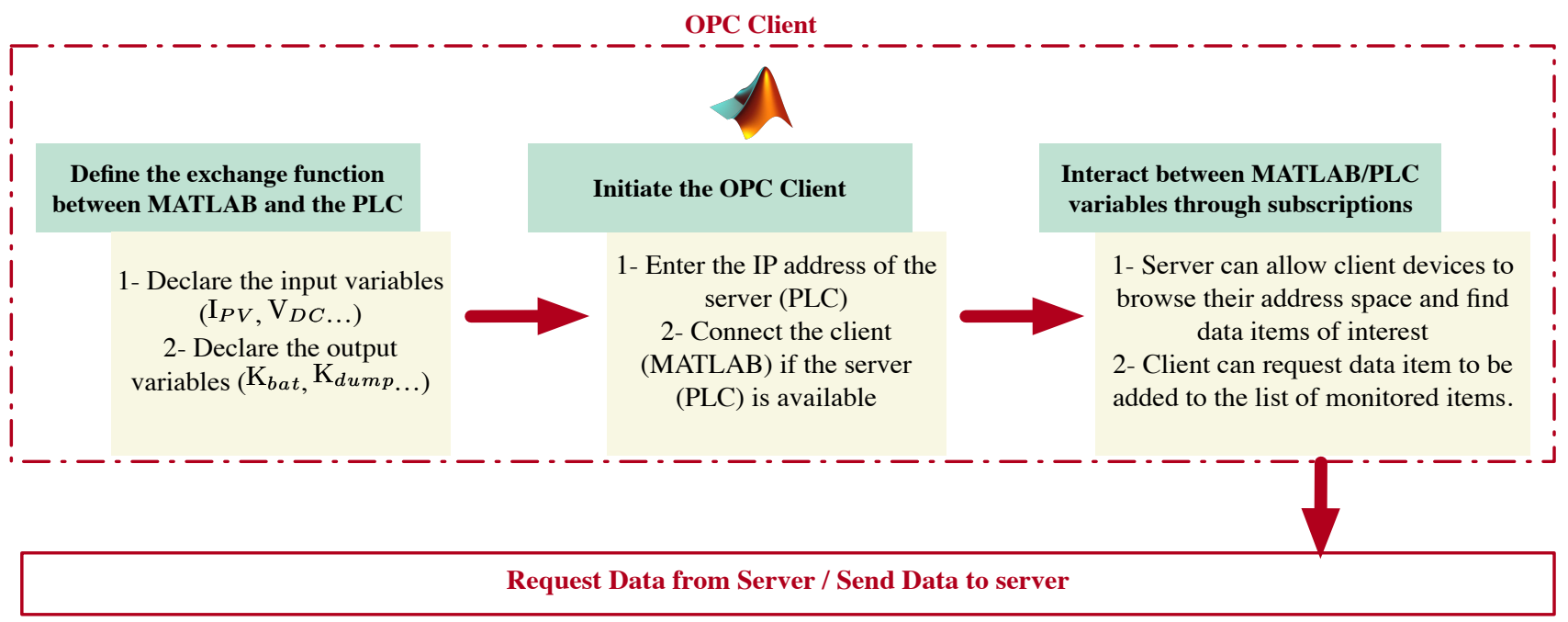

Fig. 4. The connection process between the client and the server.

The virtual PLC is connected to the workstation using the TCP/IP communication protocol, which allows remote access to the PLC using the IP address associated with the PLC. The

framework developed in this paper can share data over the wide area network (WAN) using a web server-based architecture.

An OPC Server is firstly initiated using the OPC UA library as descried in Fig. 4. Then a script is developed in order to $\mathrm{read} / \mathrm{write}$ data from the OPC Client as illustrated in

Fig. 5.

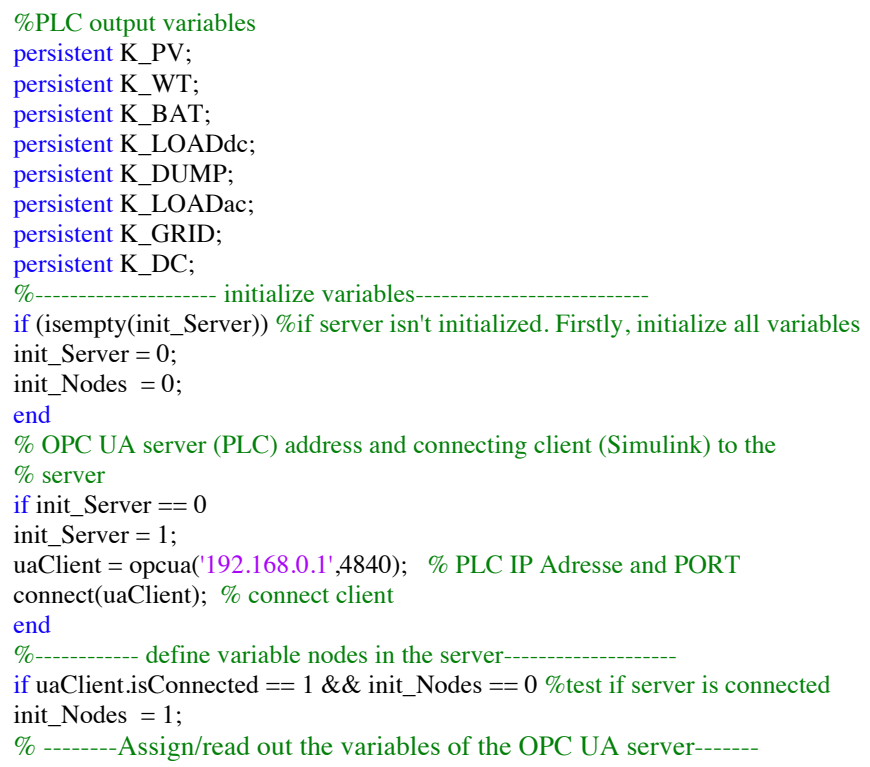




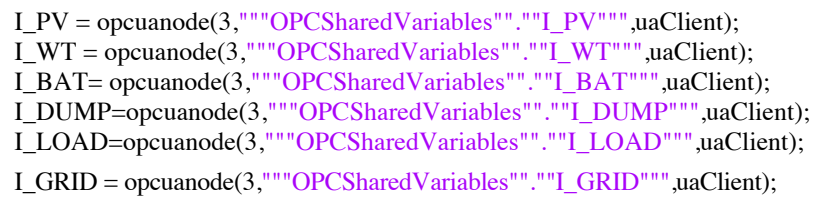

Fig. 5. An excerpt from the script describing the communication initiation of OPC-UA.

2) Establishment of PLC-HMI connection

PLC and HMI communicate through the PROFINET standard, it is an open communication protocol for industrial ethernets built upon the Standard Ethernet network and was proposed as a communication standard for industrial automation [25]. Once all data has been collected and compiled by the PLC using the OPC server, the HMI will arrange a graphical presentation that informs the operator of the system's operational status as illustrated in Fig. 15.

\section{VALIDATION: ENERGY MANAGEMENT SYSTEM IMPLEMENTATION}

The electrical layout of the system is presented in Fig. 6. The microgrid (MG) is represented as a combination of the

following elements:

- Photovoltaic cells (PV);

- Wind Turbine (WT) generator;

- Batteries;

- Common DC bus;

- Dump load;

- Loads.

The current and voltage sensors of the power generators, the state of charge and the battery temperature are connected to the PLC inputs. Although the PLC outputs control the switches $K_{\text {bat }}, K_{d u m p}$ and $K_{\text {loads }}$.

\section{Microgrid}

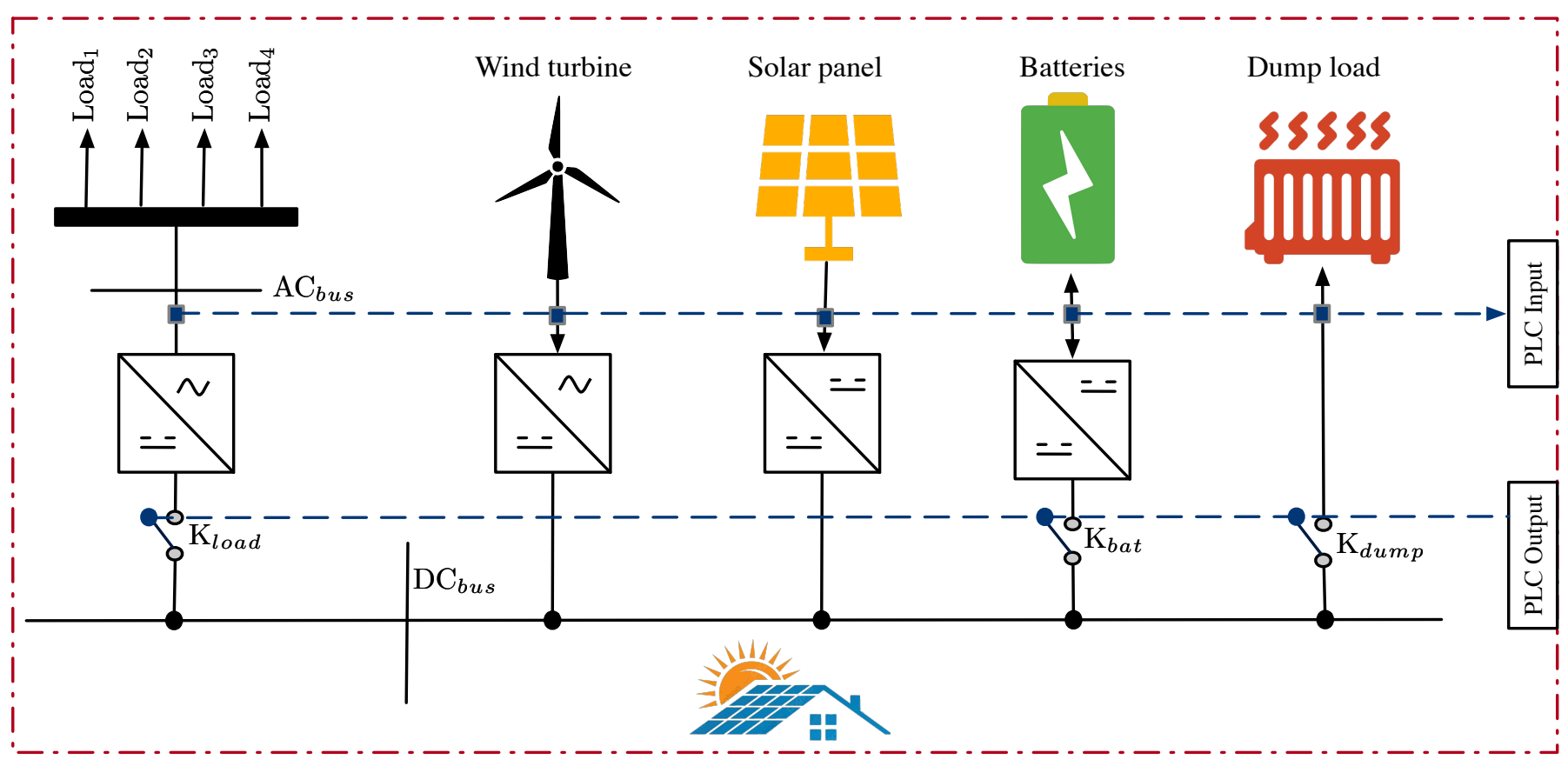

Fig. 6. Electrical architecture of the studied microgrid.

\section{A. Fuzzy-PID implementation}

The PID instruction, implemented in S7-1214 PLC with TIA PORTAL programming software, controls the DC bus by sending an output signal to the battery converter. The developed setup screen provides access to the PID parameters illustrated in Fig. 15.

The PID controller is tuned in order to achieve conventional objectives such as minimum steady-state error, stability under unpredictable climate conditions and fast system response to sudden load changes. Fig. 7 shows the microgrid control without the incorporation of an EMS. The DC voltage reference $\left(V_{d c r e f}\right)$ issued from the HMI is compared with the actual DC voltage $\left(V_{d c}\right)$ issued from the PLC. After regulating the voltage, $I_{\text {batref }}$ is compared with the actual battery current $\left(I_{b a t}\right)$ measured by the PLC and the error is processed through a PI controller. The current regulation loop provides $D_{b a t}$ signals to the PLC output which controls the battery converter. 


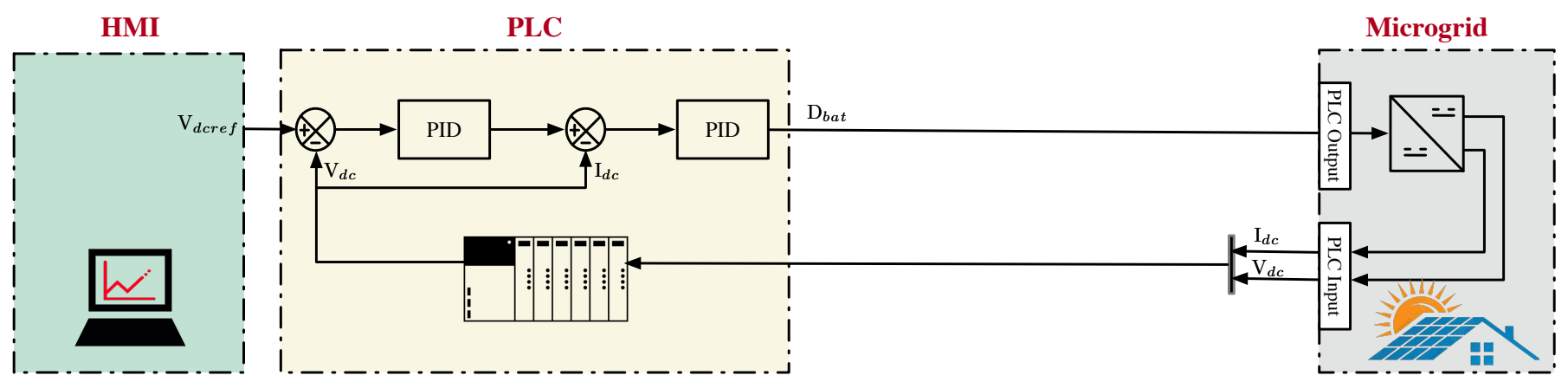

Fig. 7. Microgrid control without the incorporation of an EMS.

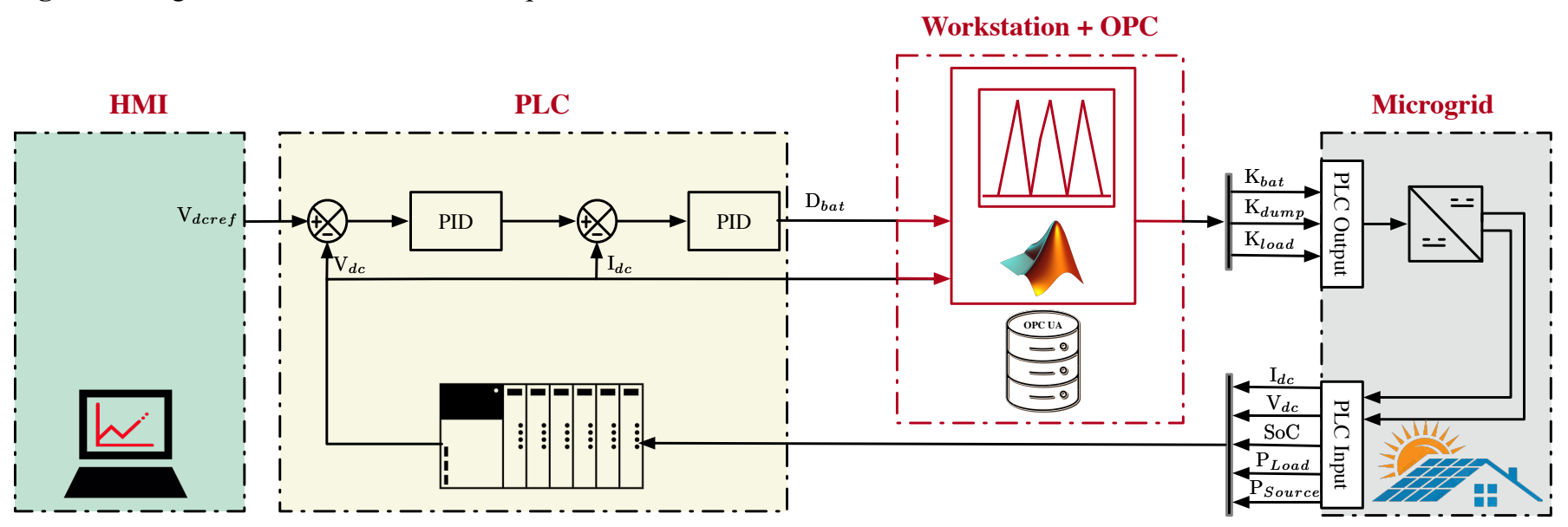

Fig. 8. Microgrid control with the incorporation of an EMS.

In order to manage the energy flow in the microgrid, a fuzzy logic-based EMS is implemented. Knowing that complex AI algorithms are not supported by PLCs, the framework developed in this paper can be used to process the algorithm under MATLAB and transfer results to the PLC through an OPC server. Fig. 8 describes the control strategy

implementation when an EMS is present.

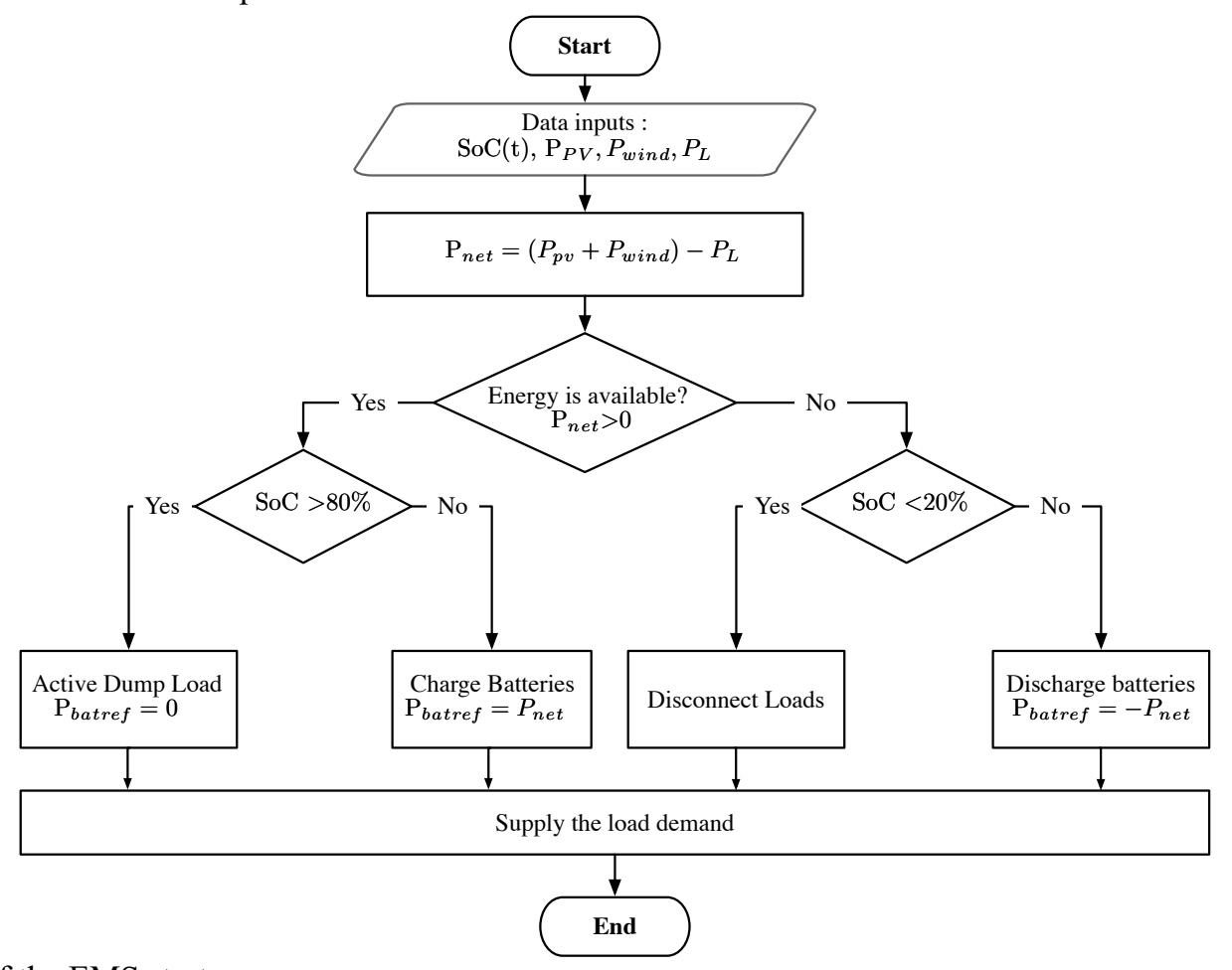

Fig. 9. Flowchart of the EMS strategy. 
The EMS algorithm is outlined in Fig. 9. Maintaining $V_{d c}$ at its reference value is obtained by managing batteries and dump load power. The batteries provide the lack of energy (high load demand). Otherwise, the energy surplus (low load demand) is used to charge the batteries within specified limits $(20 \%<S o C<80 \%)$. In case of lower demand, if the batteries are fully charged ( $\mathrm{SoC}>80 \%$ ), the power excess is dumped. Otherwise, the loads are disconnected. A bidirectional converter links batteries with the common DC bus. Through $\mathrm{K}_{\mathrm{dump}}$ switch, the dump load is used to dissipate power excess during fault or overgeneration conditions. The excess power can be converted through a heat load.

The Net power that is calculated according to (1):

$$
P_{\text {net }}=\left(P_{p v}+P_{\text {wind }}\right)-P_{\text {load }}
$$

where, $P_{\text {wind }}$ is the power generated by the wind energy system, $P_{p v}$ is the power generated by the PV energy conversion system and $P_{\text {load }}$ represents the load power demand.

\section{B. Results and validation}

The PLC integrating the PID controller is simulated using PLCSim software. The microgrid and the EMS based on fuzzy logic are simulated using MATLAB. As shown in Fig. 10, the developed framework operates according to the following steps:

1. Initialization of the OPC-UA server,

2. Simulation of the microgrid (PV, WT, battery and dump load, etc.) under variable weather conditions,

3. Acquisition of data from PLC,

4. Execution of the EMS based fuzzy logic,

5. Sending control signals to the PLCSim controller,

6. Visualization of the data by HMI.

1

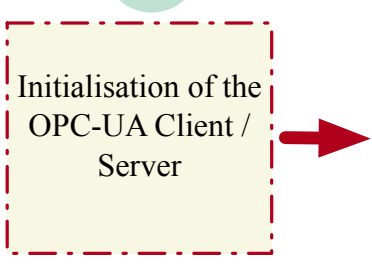

6

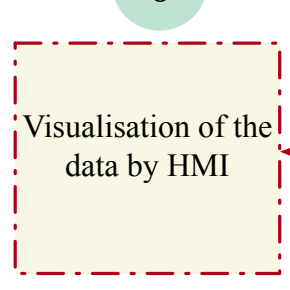

2

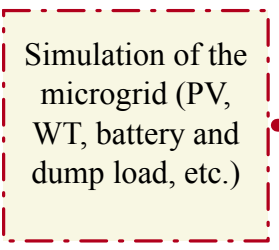

5
3

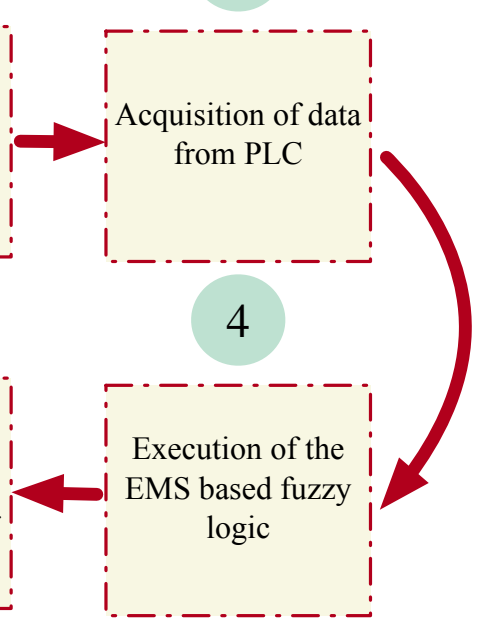

Fig. 10. The execution steps of the algorithm according to the developed framework.

1) FLC implementation results

The behavior of the MG under fluctuating climatic conditions (wind, irradiation and temperature) is considered to assess the effectiveness of the FL controller. Subsequently, the DC bus stability is evaluated under different conditions (extreme load demand, sudden climatic variation, etc.) from a voltage regulation point of view.

Fig. 11 represents the solar irradiation and the temperature

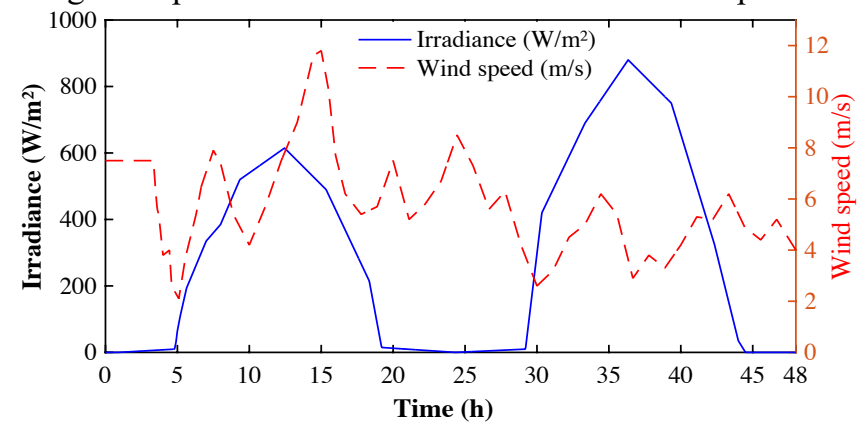

Fig. 11. Two days irradiance and wind speed profile.

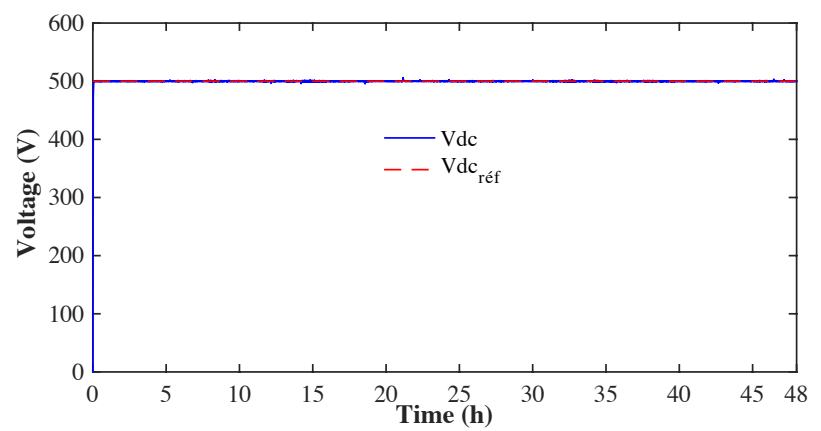

Fig. 12. DC bus voltage. 


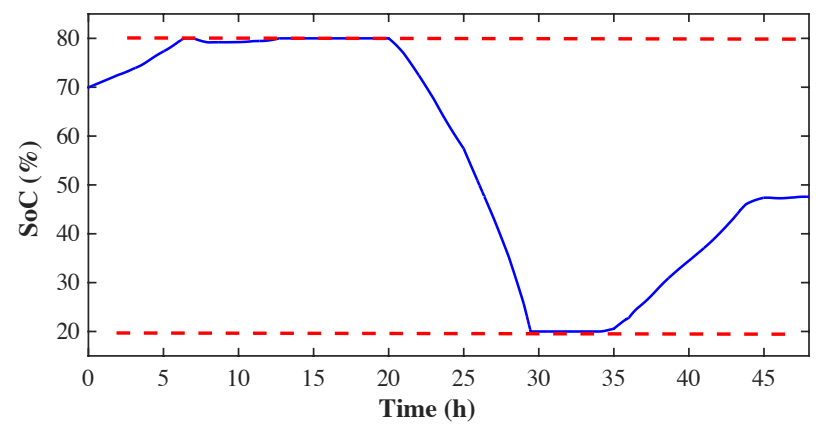

Fig. 13. Battery SoC during excess energy case.

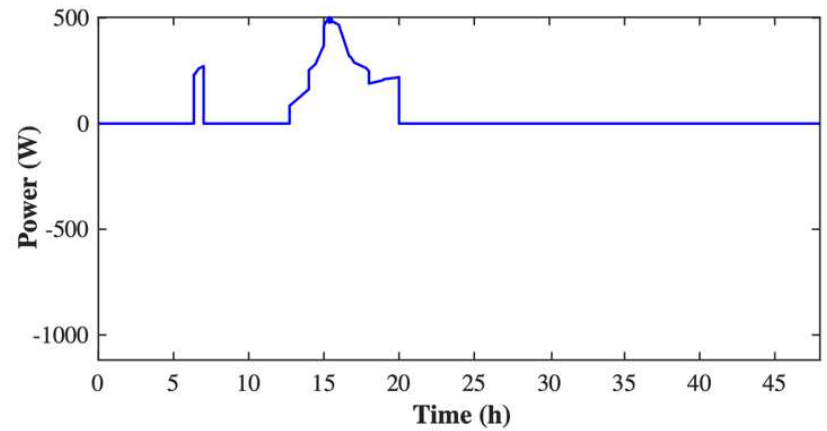

Fig. 14. Power consumed by dump load.

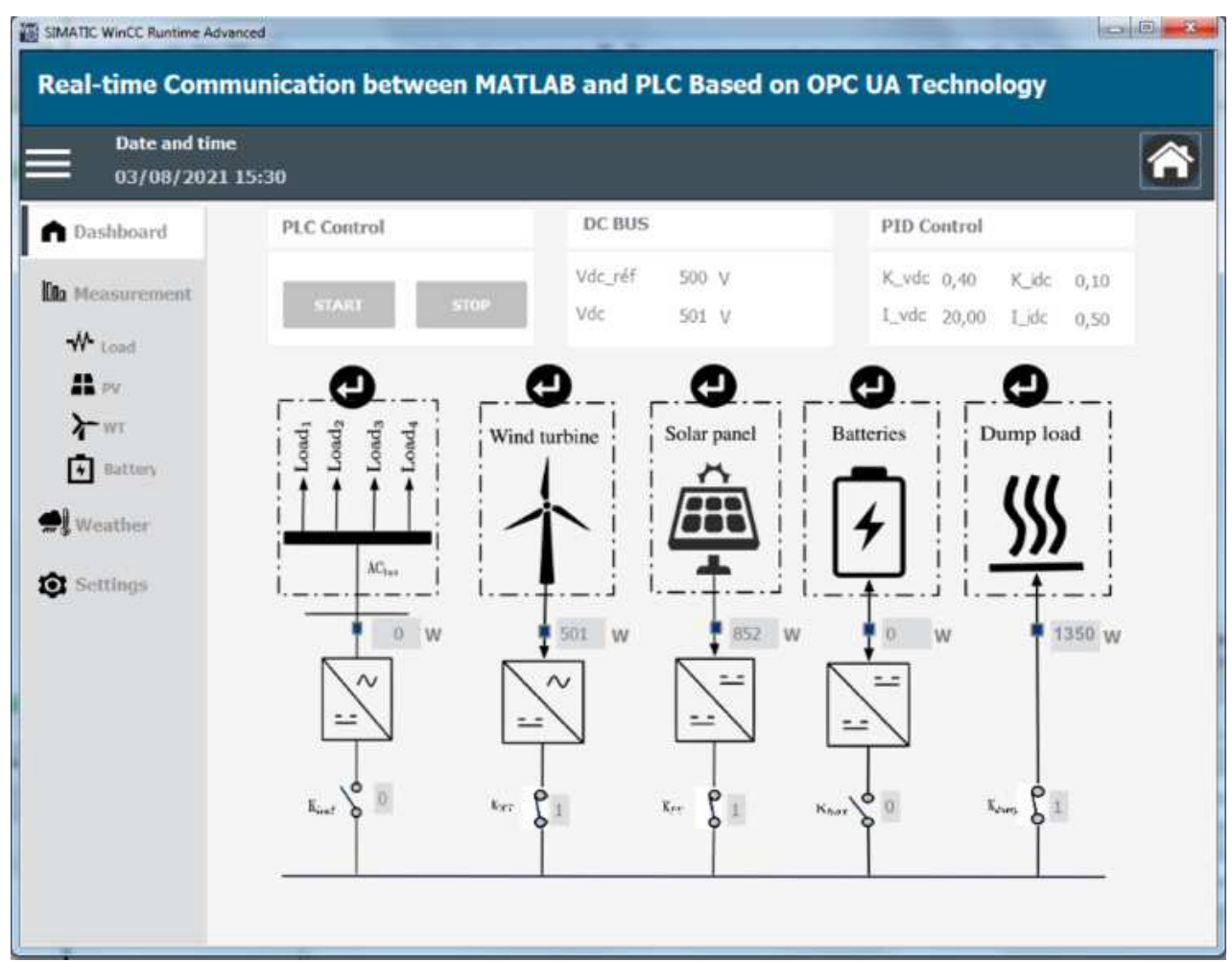

Fig. 15. Switches state during the excess energy case.

variation, respectively. Fig. 12 illustrates the evolution of the DC bus voltage during the simulation. It is constant and follows the reference voltage perfectly throughout the simulation.

2) HMI implementation results

Fig. 13 and Fig. 14 show the $\mathrm{SoC}$ and the power injected into the dump load, respectively. From $12 \mathrm{~h}$ to $20 \mathrm{~h}$, the SoC reaches the maximum limit set at $80 \%$. However, to extend their lifespan, the $E M S$ disconnects the batteries by turning off the switch $K_{b a t}$. At the same time, EMS connects the dump load to dissipate excess energy as shown in Fig. 15.

The data collected from the different sensors of the microgrid are aggregated to elaborate the view shown in Fig. 16. During the simulation, the temperature is $8^{\circ} \mathrm{C}$ and the wind blows at a speed of $7 \mathrm{~m} / \mathrm{s}$. 


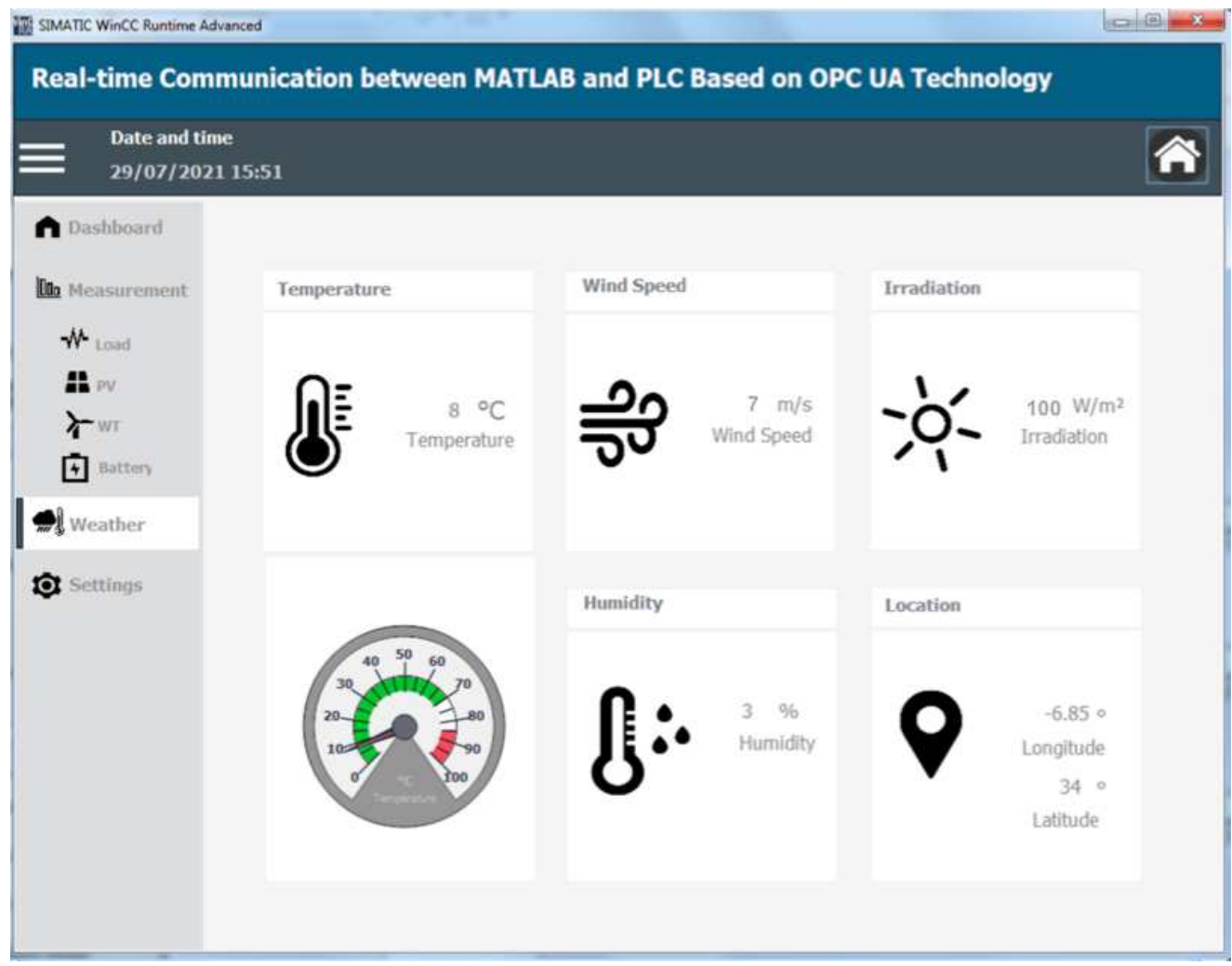

Fig. 16. Climatic condition view.

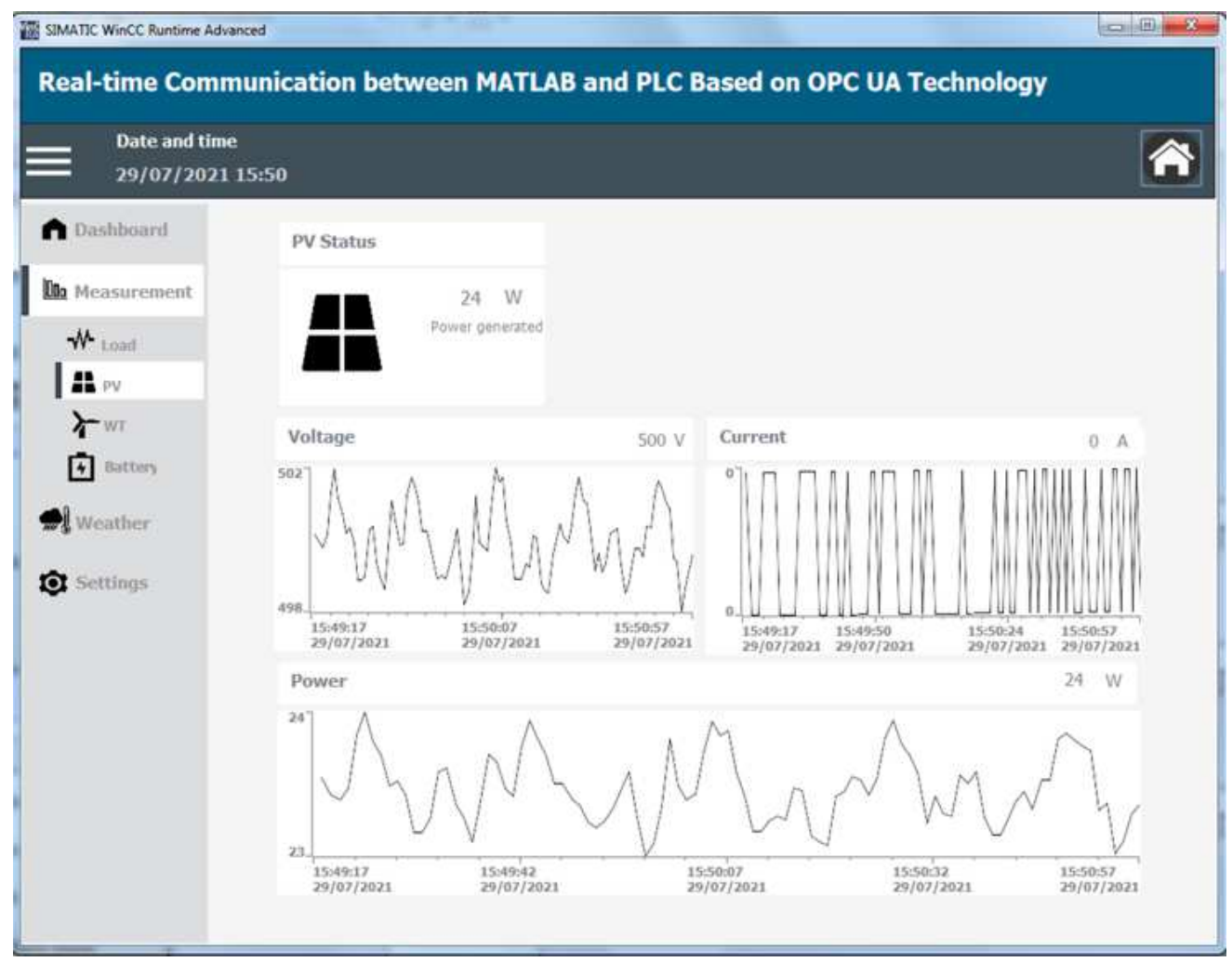

Fig. 17. PV generation view.

Fig. 17 and Fig. 18 shows PV and wind generator data respectively. The power extracted depends on climatic conditions.

The view shown in Fig. 19 represents the data of the energy storage system. The behavior of the battery remains stable within an acceptable limit fixed between $20 \%$ and $80 \%$. The DC voltage is constant and follows the reference voltage perfectly throughout the simulation. 


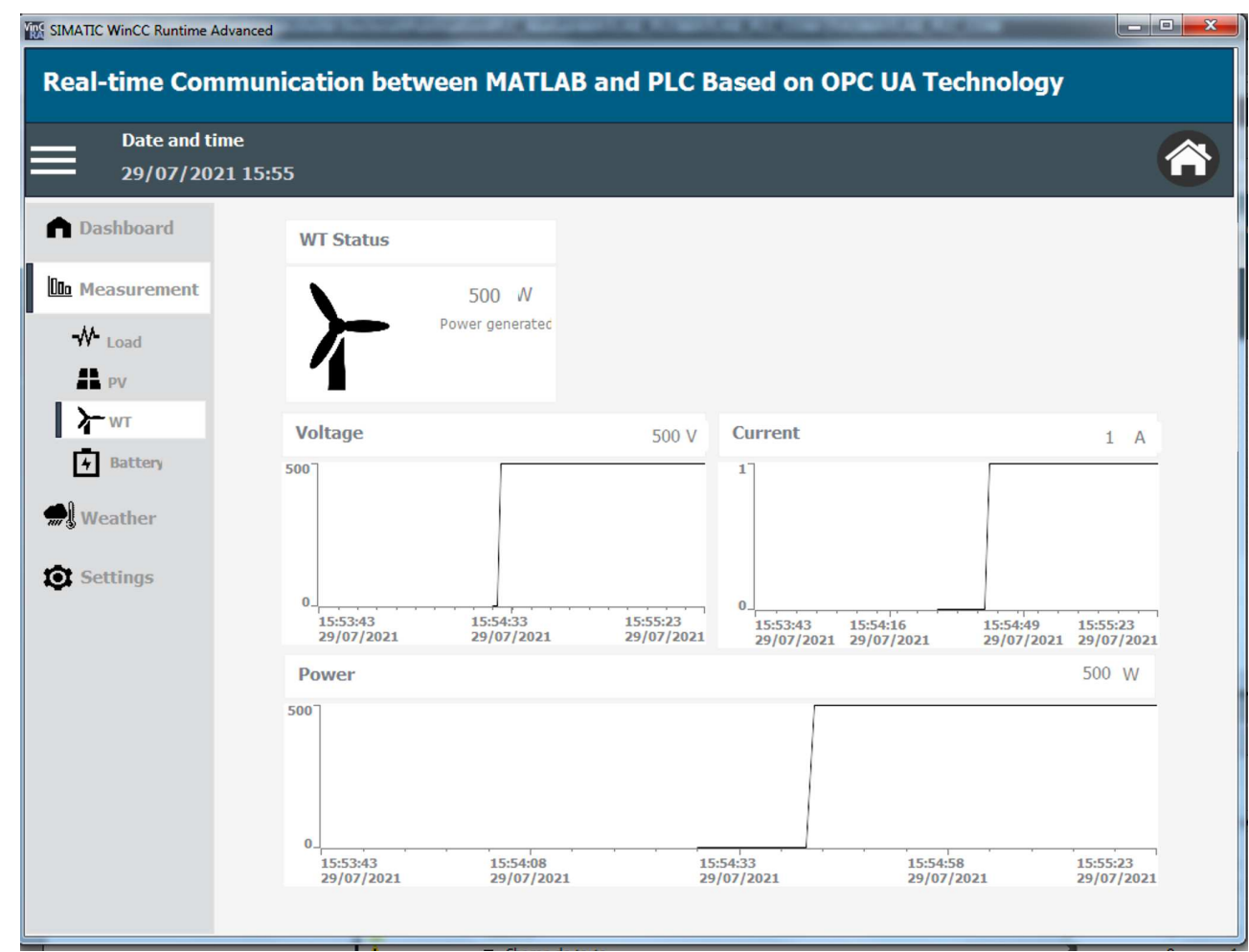

Fig. 18. Wind turbine generation view.

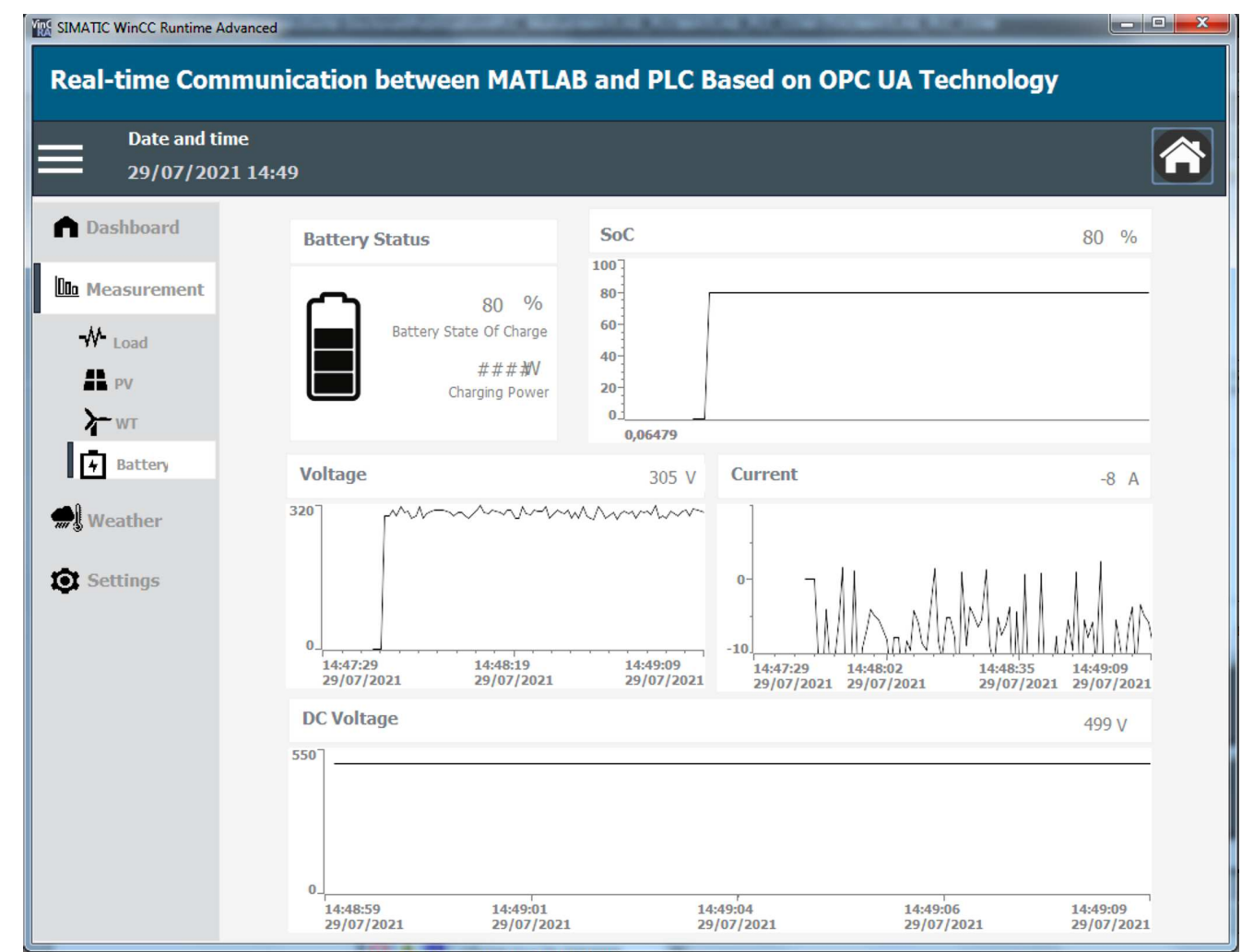

Fig. 19. Battery view.

The information indicating the daily consumption is shown in Fig. 20. The proposed system can provide a better quality of voltage and current to the consumer where the waveform and harmonic distortion of both voltage and current are satisfying.

\section{CONCLUSION}

In this paper, a Real-time simulation platform has been developed to assist in academic and research activities. The solution 


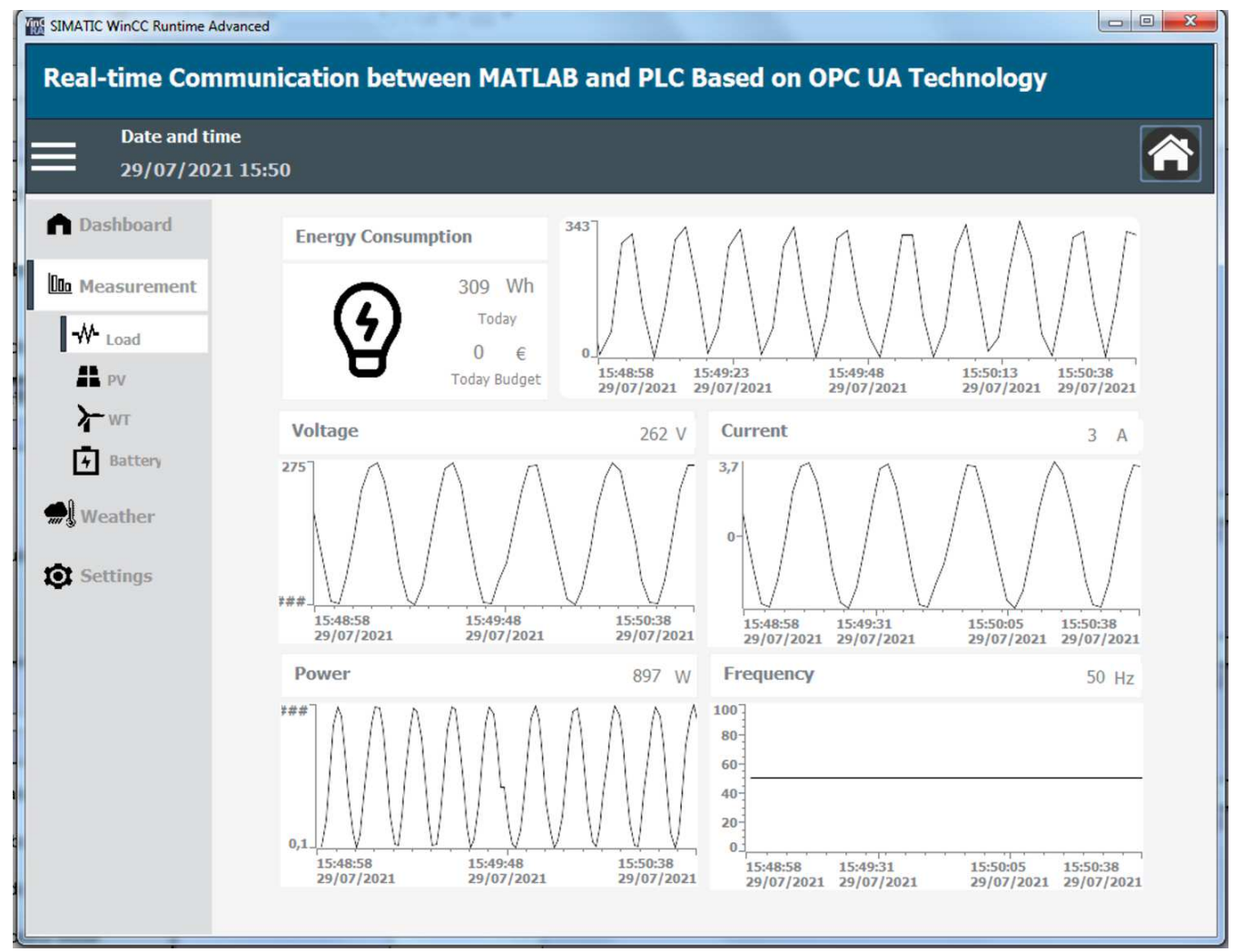

Fig. 20. Load view.

consists of using a PLC to acquire data from the

various sensors and control the system through actuators and PID controllers. Indeed, a communication framework between MATLAB and the PLC is implemented through OPC-UA protocol. As demonstrated in the obtained results, the framework can be implemented to practically test developed AI algorithms. A case study of EMS using fuzzy logic is applied to validate the results. The fuzzy logic controller development is relatively simple under MATLAB/Simulink thanks to the power of this simulation tool. Afterwards, the FL controller output signals are

transferred to the PLC for execution through OPC-UA technology. Using the platform designed in this paper, researchers can develop their complex algorithm using traditional toolboxes and libraries under MATLAB/Simulink, then it can be automatically converted to the PLC to be practically performed in real time.

OPC-UA protocol. As demonstrated in the obtained results, the framework can be implemented to practically test developed AI algorithms. A case study of EMS using fuzzy logic is applied to validate the results. The fuzzy logic controller development is relatively simple under MATLAB/Simulink thanks to the power of this simulation tool. Afterwards, the FL controller output signals are transferred to the PLC for execution through OPC-UA technology. Using the platform designed in this paper, researchers can develop their complex algorithm using traditional toolboxes and libraries under MATLAB/Simulink, then it can be automatically converted to the PLC to be practically performed in real time.

\section{REFERENCES}

[1] H. K. Ringkjøb, P. M. Haugan, and I. M. Solbrekke, "A review of modelling tools for energy and electricity systems with large shares of variable renewables," Renew. Sustain. Energy Rev., vol. 96, pp. 440-459, Nov. 2018.

[2] N. Collier and A. Kaw, "On Comparing Computational Systems - Maple, Mathcad, Mathematica \& Matlab," Comput. Educ. J., vol. 14, no. 1, Jan. 2004, Accessed: Aug. 05, 2021. [Online]. Available: https://digitalcommons.usf.edu/egr_facpub/171.

[3] L. Olatomiwa, S. Mekhilef, M. S. Ismail, and M. Moghavvemi, "Energy management strategies in hybrid renewable energy systems: A review," Renew. Sustain. Energy Rev., vol. 62, pp. 821-835, 2016.

[4] S. S. Ali and B. J. Choi, "State-of-the-Art Artificial Intelligence Techniques for Distributed Smart Grids: A Review," Electron. 2020, Vol. 9, Page 1030, vol. 9, no. 6, p. 1030, Jun. 2020.

[5] K. P. Seng, P. J. Lee, and L. M. Ang, “Embedded Intelligence on FPGA: Survey, Applications and Challenges,” Electron. 2021, Vol. 10, Page 895, vol. 10, no. 8, p. 895, Apr. 2021.

[6] H. He, R. Xiong, K. Zhao, and Z. Liu, "Energy management strategy research on a hybrid power system by hardware-in-loop experiments," Appl. Energy, vol. 112, pp. 1311-1317, Dec. 2013.

[7] T. Atalik et al., "Multi-DSP and -FPGA-based fully digital control system for cascaded multilevel converters used in FACTS applications," IEEE Trans. Ind. Informatics, vol. 8, no. 3, pp. 511-527, 2012. 
[8] M. A. Sehr et al., "Programmable Logic Controllers in the Context of Industry 4.0," IEEE Trans. Ind. Informatics, vol. 17, no. 5, pp. 3523-3533, May 2021.

[9] L. C. Tasca, E. Pignaton de Freitas, and F. R. Wagner, "A study on the performance impact of programmable logic controllers based on enhanced architecture and organization," Microprocess. Microsyst., vol. 76, p. 103082, Jul. 2020.

[10] N. Mohammed and A. M. Saif, "Programmable logic controller based lithium-ion battery management system for accurate state of charge estimation," Comput. Electr. Eng., vol. 93, p. 107306, Jul. 2021.

[11] “OPC Foundation.” https://opcfoundation.org/ (accessed Aug. 05, 2021).

[12] "OPC UA Users and Experts-Conveying Knowledge and Experience 4.0 Industrie IoT M2M," Accessed: Aug. 05, 2021. [Online]. Available: https://opcfoundation.org/webinars.

[13] S. Lehnhoff, S. Rohjans, M. Uslar, and W. Mahnke, "OPC unified architecture: A service-oriented architecture for smart grids," 2012 1st Int. Work. Softw. Eng. Challenges Smart Grid, SE-SmartGrids 2012 - Proc., pp. 1-7, 2012.

[14] M. H. Schwarz and J. Borcsok, "A survey on OPC and OPC-UA: About the standard, developments and investigations," 2013 24th Int. Conf. Information, Commun. Autom. Technol. ICAT 2013, 2013.

[15] "Legal information OPC UA methods for the SIMATIC S7-1500 OPC UA server Legal information Use of application examples," 2018, Accessed: Aug. 05, 2021. [Online]. Available: https://www.siemens.com/industrialsecurity.

[16] “OPC UA security analysis | Kaspersky ICS CERT.” https://ics-cert.kaspersky.com/reports/2018/05/10/opc-ua-security-analysis/ (accessed Aug. 05, 2021).

[17] A. Lakshmi Sangeetha, B. Naveenkumar, A. Balaji Ganesh, and N. Bharathi, "Experimental validation of PID based cascade control system through SCADAPLC-OPC and internet architectures," Measurement, vol. 45, no. 4, pp. 643-649, May 2012.

[18] F. Fernandes, M. Silva, P. Faria, Z. Vale, C. Ramos, and H. Morais, "Real-time simulation of energy management in a domestic consumer," 2013 4th IEEE/PES Innov. Smart Grid Technol. Eur. ISGT Eur. 2013, 2013.

[19] G. Ensermu, A. Bhattacharya, and N. Panigrahy, "Real-Time Simulation of Smart DC Microgrid with Decentralized Control System Under Source Disturbances,” Arab. J. Sci. Eng. 2019 448, vol. 44, no. 8, pp. 7173-7185, Apr. 2019.

[20] “OPC Toolbox Documentation.” https://www.mathworks.com/help/opc/ (accessed Aug. 05, 2021).

[21] “Totally Integrated Automation Portal | Automation Software | Siemens Global." https://new.siemens.com/global/en/products/automation/industrysoftware/automation-software/tia-portal.html (accessed Aug. 05, 2021).

[22] "IEC 61131-3:2013 | IEC Webstore | water automation, water management, smart city." https://webstore.iec.ch/publication/4552 (accessed Aug. 05, 2021).

[23] "SIMATIC HMI WinCC WinCC Runtime Professional Readme System Manual," 2017.

[24] H. .\& M. Hinchey, "Model-Based verification of embedded software.," Irish Softw. Eng. Res. Cent., 2009.

[25] "IEC 61158-1, Overview and guidance for PROFINET specifications." https://www.profibus.com/download/profinet-specification (accessed Aug. 05, 2021). 